\title{
The Breather-Like and Rational Solutions for the Integrable Kadomtsev-Petviashvili-Based System
}

\author{
Chuanjian Wang, ${ }^{1}$ Zhengde Dai, ${ }^{2}$ and Changfu Liu ${ }^{3}$ \\ ${ }^{1}$ School of Science, Kunming University of Science and Technology, Kunming 650500, China \\ ${ }^{2}$ School of Mathematics and Statistics, Yunnan University, Kunming 650091, China \\ ${ }^{3}$ School of Mathematics, Wenshan University, Wenshan 663000, China \\ Correspondence should be addressed to Chuanjian Wang; wcj20082002@aliyun.com
}

Received 12 February 2015; Revised 10 May 2015; Accepted 7 June 2015

Academic Editor: Ricardo Weder

Copyright (C) 2015 Chuanjian Wang et al. This is an open access article distributed under the Creative Commons Attribution License, which permits unrestricted use, distribution, and reproduction in any medium, provided the original work is properly cited.

\begin{abstract}
The integrable Kadomtsev-Petviashvili-based system is studied. The breather-like (a pulsating mode) and rational solutions are presented applying Hirota bilinear method and Taylor series. The intricate structures of the rational solitary wave solution are discussed mathematically and graphically. The existence conditions of three different solitary wave solution structure for the shortwave field are given by the theory of extreme value analysis. By controlling the wave number of the background plane wave we may control the the behavior of rational solitary wave. However, the shape of the rational solitary wave solution for the real long-wave field is not affected as the wave number is varied.
\end{abstract}

\section{Introduction}

The integrable Kadomtsev-Petviashvili-based system is derived from the Kadomtsev-Petviashvili (KP) equation via an asymptotically exact reduction method based on the Fourier expansion and spatiotemporal rescaling [1]; it can be written in the following form:

$$
\begin{aligned}
i u_{t}+u_{x x}+u v & =0, \\
v_{t}+v_{y}+\left(|u|^{2}\right)_{x} & =0,
\end{aligned}
$$

where $i=\sqrt{-1}, u(x, y, t)$ is a complex function of two scaled space coordinates $x, y$ and time $t$, and $v(x, y, t)$ is a real one. The subscripts $x, y$, and $t$ denote partial derivatives. The integrability property and Lax pairs of system (1) were obtained in [1]. It is a family of nonlinear partial equation that are often used to describe the phenomenon in the relevant physical fields such as nonlinear optic, plasma physics, and hydrodynamic [2]. It has been studied by many authors. For example, exact traveling wave solutions of system (1) have been obtained using the generalized algebraic method [3]. The single and combined generalized solitary wave solutions are obtained by the Exp-function method [4]. Abundant soliton and periodic wave solutions are presented by the trigonometric and hyperbolic functions ansatz [5]. Bright one- and two-soliton solutions have been gotten with the Hirota method and symbolic computation [6]. However, the integrable Kadomtsev-Petviashvili-based system still possesses many other interesting solution structures that have not been found. In this paper, by the Exp-function method with complex frequency and complex wave number, the breather-like solution (a pulsating mode) is presented. Employing the Taylor series or the long wave limits [7-11], the rational solution is obtained. Indeed, this kind of solution is also called rogue wave [8-11]. Rogue wave was first observed under circumstances of arbitrary depths of the ocean. The first-order rational solution (Peregrine solitons) of the self-focusing nonlinear Schrödinger equation was first proposed by Peregrine to describe the rogue wave phenomenon [12]. One always has two or even more times higher amplitude than their surrounding waves and generally they form in a short time. They are localized in both time and space and decays algebraically to the background plane wave. In several other physical fields [13], rogue wave has drawn much attention such as oceanography, atmospherics, nonlinear optics, and Bose-Einstein condensates. Many other 
evolution equations exhibit rogue wave solutions, for example, the focusing and defocusing Ablowitz-Ladik equations [14], Davey-Stewartson equation [15], coupled SchrödingerBoussinesq equation [16], Sasa-Satsuma equation [17], coupled nonlinear Schrödinger and Maxwell-Bloch equations [18], systems displaying PT-symmetry [19], three-component coupled nonlinear Schrödinger equation [20], and even modeling in finance [21].

\section{Breather-Like and Rational Solutions}

In this section, we will use Hirota technique [22] and Taylor series to construct the breather-like (a pulsating mode) and rational solutions of system (1).

2.1. Hirota Technique and Taylor Series Method. First, by the dependent variable transformation

$$
\begin{aligned}
& u=\frac{G}{F}, \\
& v=2(\ln F)_{x x},
\end{aligned}
$$

system (1) can be transformed into the Hirota's bilinear form

$$
\begin{array}{r}
\left(i D_{t}+D_{x}^{2}\right) G \cdot F=0 \\
\left(D_{t} D_{x}+D_{y} D_{x}-A\right) F \cdot F+G \cdot G^{*}=0
\end{array}
$$

where the $D$-operator [22] is defined by

$$
\begin{gathered}
D_{x}^{m} D_{t}^{n} F(x, t) \cdot G(x, t)=\left(\frac{\partial}{\partial x}-\frac{\partial}{\partial x^{\prime}}\right)^{m}\left(\frac{\partial}{\partial t}-\frac{\partial}{\partial t^{\prime}}\right)^{n} \\
\left.\cdot\left[F(x, t) G\left(x^{\prime}, t^{\prime}\right)\right]\right|_{x^{\prime}=x, t^{\prime}=t}, \quad n, m \geq 0,
\end{gathered}
$$

and $G(x, y, t)$ is a complex valued function and $F(x, y, t)$ is a real, $G^{*}(x, y, t)$ is the conjugate function of $G(x, y, t)$, and $A$ represents an arbitrary constant of integration. So the solutions of original partial differential equation can be converted into the solutions of bilinear differential equations.

Second, we solve the above bilinear differential equations to get the breather-like solution using the two-soliton solution with complex frequency and complex wave number

$$
\begin{aligned}
& F=1+f_{1}+f_{2}+M f_{1} f_{2}, \\
& G=u_{0} e^{-i\left(\tau+\theta_{0}\right)}\left(1+a_{1} f_{1}+a_{2} f_{2}+M a_{1} a_{2} f_{1} f_{2}\right),
\end{aligned}
$$

where $f_{1}=e^{i p x-\Omega(t+q y)+r}, f_{2}=e^{-i p x-\Omega^{*}(t+q y)+r}$, and $\tau=k x+$ $l y+c t$, and $l, k, c, p, q, M, u_{0}$ are real, $a_{1}, a_{2}, \Omega$ are complex, and $r$ is an arbitrary phase parameter, and $*$ represents the complex conjugate.

Third, using the Taylor series of the functions $F$ and $G$ at the point $p=0$

$$
\begin{aligned}
F(p)= & F(0)+p F^{\prime}(0)+p^{2} \frac{F^{\prime \prime}(0)}{2 !}+\cdots+p^{n} \frac{F^{(n)}(0)}{n !} \\
& +\cdots,
\end{aligned}
$$

$G$ is similar to $F$; then we can obtain the rational solution.

2.2. Breather-Like (a Pulsating Mode). After the long and tedious calculation with Hirota technique, the breather-like solution can be rewritten in terms of trigonometric and hyperbolic functions

$$
\begin{aligned}
& u=u_{0} e^{-i\left(\tau+\theta_{0}-\theta_{1}\right)} \frac{\sqrt{M} \cosh \left(\Re(\Omega)(q y+t)-\phi_{1}-r-i \theta_{1}\right)+\cos \left(p x-\mathfrak{I}(\Omega)(q y+t)-i \phi_{2}\right)}{\sqrt{M} \cosh \left(\Re(\Omega)(q y+t)-\phi_{1}-r\right)+\cos (p x-\mathfrak{I}(\Omega)(q y+t))}, \\
& v=-\frac{2 p^{2}\left(\sqrt{M} \cos (p x-\mathfrak{I}(\Omega)(q y+t)) \cosh \left(\Re(\Omega)(q y+t)-\phi_{1}-r\right)+1\right)}{\left(\sqrt{M} \cosh \left(\Re(\Omega)(q y+t)-\phi_{1}-r\right)+\cos (p x-\mathfrak{I}(\Omega)(q y+t))\right)^{2}},
\end{aligned}
$$

where these parameters $c, p, k, M, a_{1}, a_{2}$ satisfy the following relations

$$
\begin{aligned}
A & =u_{0}^{2}, \\
c & =k^{2}, \\
a_{1} & =\frac{i \Omega-2 k p-p^{2}}{i \Omega-2 k p+p^{2}}, \\
a_{2} & =\frac{i \Omega^{*}+2 k p-p^{2}}{i \Omega^{*}+2 k p+p^{2}}, \\
M & =1+\frac{p^{4}}{\mathfrak{R}^{2}(\Omega)} .
\end{aligned}
$$

And the complex number $\Omega$ is given as follows:

$$
2 p^{3} u_{0}^{2}=i \Omega\left(i \Omega-2 k p+p^{2}\right)\left(i \Omega-2 k p-p^{2}\right)(q+1) .
$$

$\mathfrak{R}(\Omega)$ represents the real part of complex $\Omega$ and $\mathfrak{\Im}(\Omega)$ represents the imaginary part of complex $\Omega$. The parameter $p$ defines the period of the solution structure, and $\phi_{1}=\ln \sqrt{M}$, $\phi_{2}=\ln \left(\left(\mathfrak{R}^{2}(\Omega)+(\mathfrak{I}(\Omega)+2 k+p)^{2}\right) /\left(\mathfrak{R}^{2}(\Omega)+(\mathfrak{I}(\Omega)+2 k-\right.\right.$ $\left.\left.p)^{2}\right)\right)^{1 / 2}$, and $\theta_{1}=\arctan \left(2 p^{2} \mathfrak{R}(\Omega) /\left((2 k p+\mathfrak{I}(\Omega))^{2}+\mathfrak{R}^{2}(\Omega)-\right.\right.$ $\left.p^{4}\right)$ ). Obviously, $M>1$, the solution (7) has no poles, and it is well behaved everywhere. It is a nonsingular solution. This solution is composed of trigonometric and hyperbolic functions. It presents a kind of interaction solutions between trigonometrical waves and exponential waves. It is periodic in $x$-direction with period $2 \pi / p$ and represents the localized 
oscillation. At the same time it is also a solitary wave solution in $y$-direction. So, this solution is a breather-like solution. In [23], it is also called complexiton solution. Some asymptotic behaviors of the obtained solution (7) can be found with $\mathfrak{R}(\Omega)>0$ :

$$
\begin{aligned}
& (u, v) \longrightarrow\left(u_{0} e^{-i\left(\tau+\theta_{0}\right)}, 0\right), \quad \text { as } t \longrightarrow+\infty ; \\
& (u, v) \longrightarrow\left(u_{0} e^{-i\left(\tau+\theta_{0}-2 \theta_{1}\right)}, 0\right), \quad \text { as } t \longrightarrow-\infty .
\end{aligned}
$$

Note that $\left(u_{0} e^{-i\left(\tau+\theta_{0}-2 \theta_{1}\right)}, 0\right)$ and $\left(u_{0} e^{-i\left(\tau+\theta_{0}\right)}, 0\right)$ are two different positions of the same fixed cycle $\left(u_{0} e^{-i \tau}, 0\right) .\left(u_{0} e^{-i \tau}, 0\right)$ is the plane wave solution to system (1). This means that, in limit $t \rightarrow \pm \infty$, the solution will approach a plane wave solution, except for some phase shift. This phenomenon shows that the obtained solution (7) is a homoclinic wave solution. We also can observe that this solution is exponentially decaying along the direction of propagation $y$.

2.3. Rational Solution (Rogue Wave). In this section, we study the rational solution from breather-like solution. Let $\Omega=$ $p \Omega_{1}$ and $r=i \pi$, considering the Taylor series of the functions $F$ and $G$ at the point $p=0$, that is, taking the long wave limits; then we obtain the following rational solution:

$$
\begin{aligned}
u= & u_{0} e^{-i\left(\tau+\theta_{0}\right)}\left(1-\frac{4\left(i\left(\left(\mathfrak{I}\left(\Omega_{1}\right)+2 k\right) x+\left(\mathfrak{R}^{2}\left(\Omega_{1}\right)-2 k \mathfrak{I}\left(\Omega_{1}\right)-\mathfrak{J}^{2}\left(\Omega_{1}\right)\right)(q y+t)\right)+1\right)}{\left(\left(x-(q y+t) \mathfrak{I}\left(\Omega_{1}\right)\right)^{2}+(q y+t)^{2} \mathfrak{R}^{2}\left(\Omega_{1}\right)+1 / \mathfrak{R}^{2}\left(\Omega_{1}\right)\right)\left|2 k-i \Omega_{1}\right|^{2}}\right), \\
v= & \frac{4}{\left(x-\mathfrak{I}\left(\Omega_{1}\right)(q y+t)\right)^{2}+\mathfrak{R}^{2}\left(\Omega_{1}\right)(q y+t)^{2}+1 / \mathfrak{R}^{2}\left(\Omega_{1}\right)} \\
& -\frac{8\left(x-\mathfrak{I}\left(\Omega_{1}\right)(q y+t)\right)^{2}}{\left(\left(x-\mathfrak{I}\left(\Omega_{1}\right)(q y+t)\right)^{2}+\mathfrak{R}^{2}\left(\Omega_{1}\right)(q y+t)^{2}+1 / \mathfrak{R}^{2}\left(\Omega_{1}\right)\right)^{2}},
\end{aligned}
$$

where $\tau=k x+l y+c t$, and $\Omega_{1}$ satisfy

$$
2 u_{0}^{2}=i \Omega_{1}\left(i \Omega_{1}-2 k\right)^{2}(q+1) .
$$

$\mathfrak{R}\left(\Omega_{1}\right)$ represents the real part of complex $\Omega_{1}$ and $\Re\left(\Omega_{1}\right) \neq 0$. $\mathfrak{I}\left(\Omega_{1}\right)$ represents the imaginary part of complex $\Omega_{1}$ and $k \neq$ $-\Im\left(\Omega_{1}\right) / 2$. It is clear that (11) is a rational solution. It is a new type of rational homoclinic wave solution; that is,

$$
(u, v) \longrightarrow\left(u_{0} e^{-i\left(\tau+\theta_{0}\right)}, 0\right), \quad \text { as } t \longrightarrow \pm \infty .
$$

It is algebraically decaying, rather than exponentially decaying. This family of solution is clearly nonsingular.

2.4. Amplitude of Rational Solution. For convenience, we assume $\Omega_{1}=n+d i$. Here $n$ and $d$ are two real parameters. First, we consider the function $u(t, x, y)$ in (11). Let $U(x, y)=$ $|u(0, x, y)|^{2} u_{0}^{-2}$. After calculating, five critical points of the function $U(x, y)$ are easily obtained:

$$
\begin{gathered}
\left\{(x, y) \mid A_{\epsilon}\left(\epsilon \frac{2(n \delta-k) \sqrt{\delta^{2}-1 / 3}}{3 n^{2}\left(\delta^{2}+1\right)}, \frac{\epsilon \sqrt{\delta^{2}-1 / 3}}{3 n^{2}\left(\delta^{2}+1\right) q}\right),\right. \\
\left.B_{\epsilon}\left(\frac{\epsilon \sqrt{3-\delta^{2}}(n-d \delta)}{n^{2}\left(\delta^{2}+1\right)},-\frac{\epsilon \sqrt{3-\delta^{2}} \delta}{n^{2}\left(\delta^{2}+1\right) q}\right), O(0,0)\right\},
\end{gathered}
$$

where $\delta=(2 k+d) / n, \epsilon= \pm 1$. From the above four critical points $A_{\epsilon}, B_{\epsilon}$. The existing condition of $A_{\epsilon}$ is $\delta^{2} \geq 1 / 3$ and $B_{\epsilon}$ is $\delta^{2} \leq 3$. Their public region is $1 / 3 \leq \delta^{2} \leq 3$. Thus, $(0,+\infty)$ can be divided into three parts by $1 / 3$ and $3:(0,1 / 3] \cup(1 / 3,3) \cup$ $[3,+\infty)$. Moreover, if $\delta^{2} \rightarrow 3$ or $1 / 3, A_{\epsilon}$ or $B_{\epsilon} \rightarrow O$. So, we only consider the critical point $O(0,0)$. After calculation, we get

$$
\begin{aligned}
S & =\left.\frac{\partial^{2}}{\partial x^{2}} U(x, y)\right|_{O(0,0)}=\frac{48\left(\delta^{2}-1\right)}{n^{2}\left(\delta^{2}+1\right)^{2}}, \\
\Delta & =\left|\begin{array}{ll}
\frac{\partial^{2}}{\partial x^{2}} U(x, y) & \frac{\partial^{2}}{\partial x \partial y} U(x, y) \\
\frac{\partial^{2}}{\partial y \partial x} U(x, y) & \frac{\partial^{2}}{\partial y^{2}} U(x, y)
\end{array}\right|_{O(0,0)} \\
& =\frac{(16 q)^{2}\left(\delta^{2}-1 / 3\right)\left(\delta^{2}-3\right)}{3 n^{2}\left(\delta^{2}+1\right)^{4}} .
\end{aligned}
$$

Applying extreme values analysis for the function $U(x, y)$, we can obtain the following results.

(1) If $\delta^{2} \in(0,1 / 3]$, then $S<0$ and $\Delta>0$. The function $U(x, y)$ has three local extreme points: $O, B_{+}$, and $B_{-} . B_{\epsilon}$ are two local minimum value points and the local minimum value is $U_{B_{\varepsilon}}=0 . O(0,0)$ is the only local maximum point of the function $U(x, y)$ and the local maximum value is $U_{O}=\left(4 /\left(1+\delta^{2}\right)-1\right)^{2}$. Clearly $U_{\mathrm{O}}\left(\delta^{2}\right)$ is monotonically decreasing with $\delta^{2} \epsilon$ $(0,1 / 3)$. So at local maximum point, if $\delta \rightarrow 0$, the maximum amplitude of the function $U(x, y) \rightarrow 9$; this shows that the amplitude of the rational solitary wave is three times that of the background plane wave. From Figure 1(a), we can see that the profile is a single hump structure. It a typical Peregrine-soliton-like structure-a hump and two holes, two holes separated by a hump. It is a bright solitary wave solution [12]. 


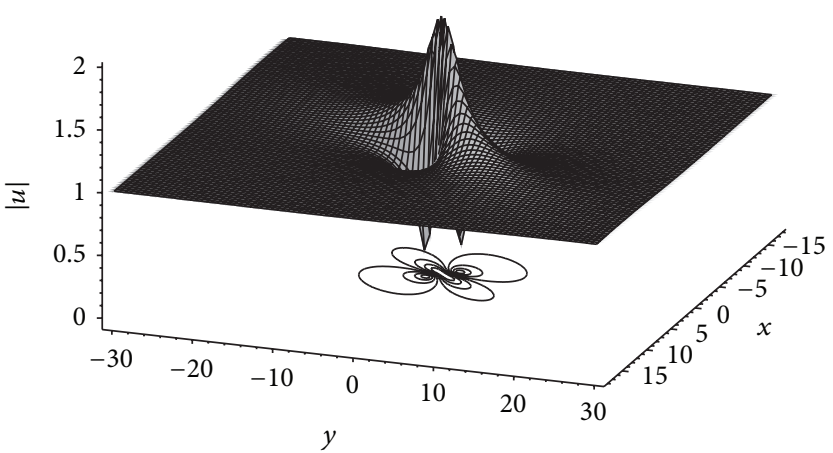

(a)

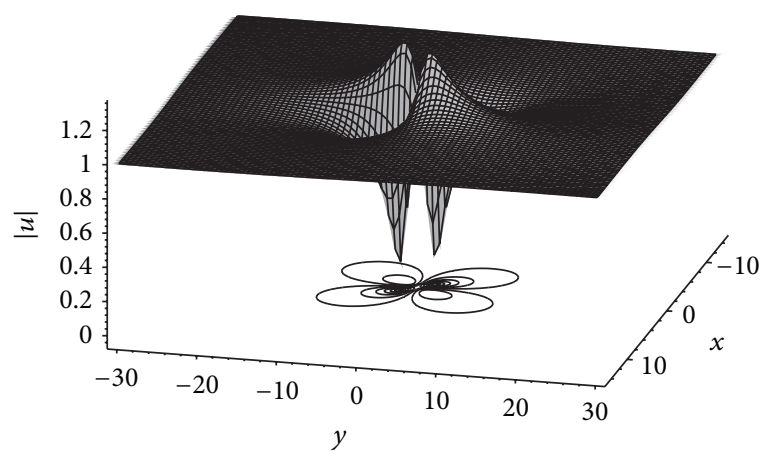

(b)

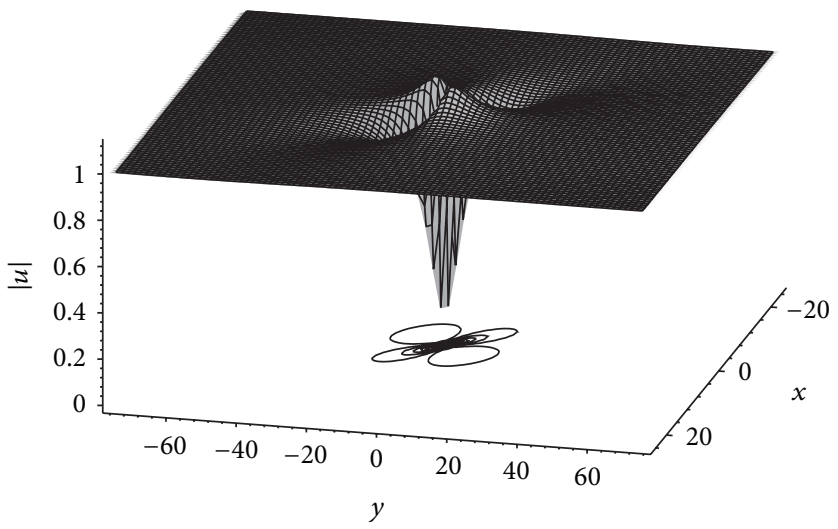

(c)

Figure 1: Spatial structures of the rational solution (11): (a) $k=0$, (b) $k=0.5$, (c) $k=0.8$ and $u_{0}=l=1$, and $q=1 / 2$. Curved lines drawn at the bottom of this figure are contour lines.

(2) If $\delta^{2} \in(1 / 3,3)$, then $S<0$ or $>0$ and $\Delta \leq 0$. The function $U(x, y)$ has five critical points: $O, B_{\epsilon}$, and $A_{\epsilon}$. The point $\mathrm{O}(0,0)$ is not a local extremum point. Similarly, we can judge that the function $U(x, y)$ has two local maximum points $A_{+}$and $A_{-}$and two local minimum points $B_{+}$and $B_{-}$. The local maximum value is $U_{A_{\epsilon}}=1+1 / \delta^{2}$ and the local minimum value is $U_{B_{\epsilon}}=0$. Obviously, $U_{B_{\epsilon}}<U_{O}<U_{A_{\epsilon}}$. If $\delta \rightarrow 1 / 3$, the maximum amplitude of the function $U(x, y) \rightarrow$ 4 ; this shows that the amplitude of the rational solitary wave is two times that of the background plane wave. Since near the origin the figure has the shape of a saddle, point $O(0,0)$ is a saddle point (see Figure 1(b)). Figure 1(b) also shows that the rational solitary wave solution $U(x, y)$ has four lumps, two upper hills and two down holes. The contour lines are like a four-petaled flower. It is quite different from Figure 1(a). Comparing with Figure 1(a), we can also think that the single hump is split into two smaller lumps, forming a four lumps pattern. The amplitude becomes smaller.

(3) If $\delta^{2} \in[3,+\infty)$, then $S>0$ and $\Delta>0$. The function $U(x, y)$ has three local extreme points: $O, A_{+}$, and $A_{-} . \mathrm{O}(0,0)$ is the only local minimum point of the function $U(x, y)$ and the local minimum value is $U_{O}=\left(4 /\left(1+\delta^{2}\right)-1\right)^{2}$. The local maximum value is
$U_{A_{c}}=1+1 / \delta^{2}$ at points $A_{\epsilon}$. From Figure $1(\mathrm{c})$, we can see that there is a deep hole under the plane wave. On the the background plane wave, two small lumps were separated by this deep hole. It is a dark solitary wave solution [2]. Comparing with Figure 1(b), two dark solitary wave solutions combine into a dark solitary wave solution.

The analysis above indicates that the dynamics of rational solution $u(t, x, y)$ depend on the value of the parameter $\delta$. The points $1 / 3$ and 3 are two critical points. The behavior of rational solution $u(t, x, y)$ changes suddenly as the parameter $\delta$ crosses the critical point $1 / 3$ or 3 . Indeed, $\delta=(2 k+$ $d) / n$ and $k$ is the wave number of the background plane wave. When $\delta^{2}=1 / 3$ or 3 , the wave number $k$ is $-(3 d \mp$ $\sqrt{3} n) / 6$ and $-(d \mp \sqrt{3} n) / 2$, respectively. So the behavior of $u(t, x, y)$ will change suddenly as the wave number $k$ is varied; then it is said to have undergone a bifurcation [24]. At a point of bifurcation, stability will be lost. It shows that the structure of the rational solution is instability. At the same time, we may control the behavior of rational rogue wave by controlling the wave number of the background plane wave. The Nizhnik-Novikov-Veselov equation [25] also exhibits intriguing structures regarding solitary wave.

Second, considering the real long-wave function $v(x, y, t)$ in (11), we can find that the profile does not vary too much in shape. Let $V(x, y)=v(0, x, y)$. After calculating, we can 


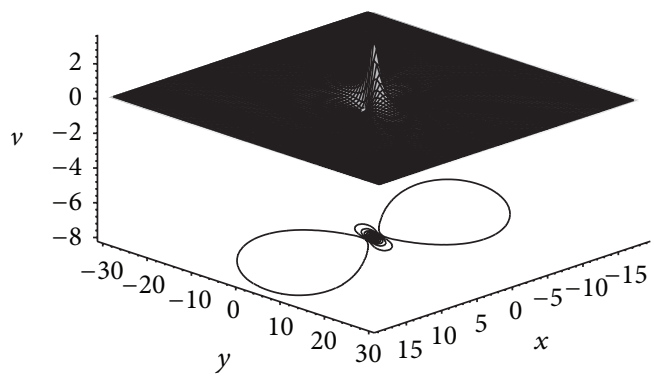

(a)

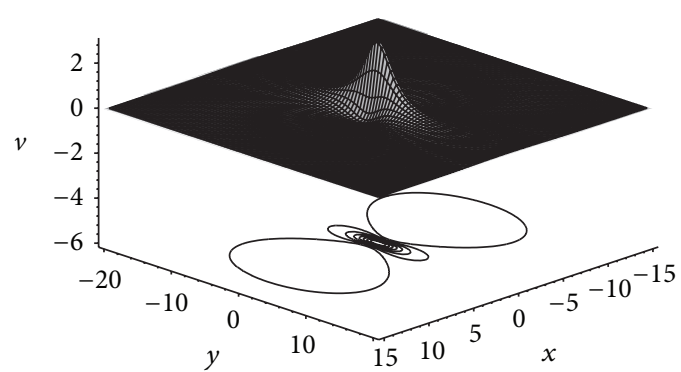

(b)

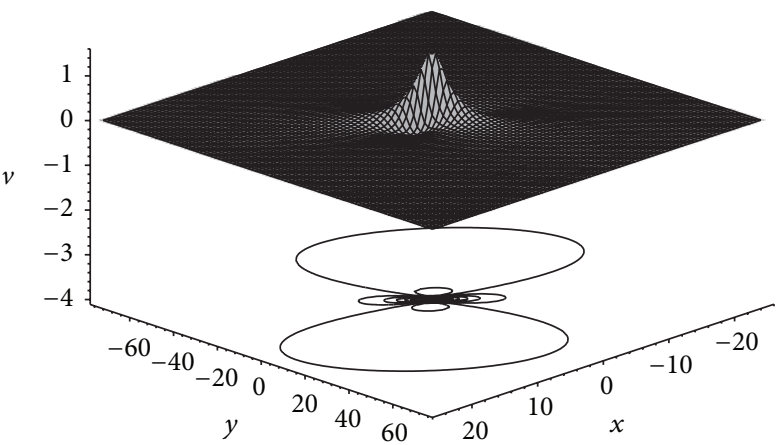

(c)

FIgure 2: Spatial structures of the rational solution (11): (a) $k=0$, (b) $k=0.5$, (c) $k=0.8$ and $u_{0}=l=1$, and $q=1 / 2$. Curved lines drawn at the bottom of this figure are contour lines.

obtain easily that the function $V(x, y)$ has three extreme value points: $O(0,0), D_{ \pm}( \pm \sqrt{3} / n, 0)$. At the point $O(0,0)$, the value of the function $V(x, y)$ is equal to $V_{O}=4 n^{2}$, and the value of the function $V(x, y)$ is $V_{D_{ \pm}}=-n^{2} / 2$ at the two points $D_{+}$. By a similar extreme value analysis, the point $O(0,0)$ is the only local maximum value point of the function $V(x, y) . D_{+}$and $D_{-}$are two local maximum value points of the function $V(x, y)$. The amplitude of the rational solution $v(x, y, t)$ depends on the parameter $n$. Thus, by controlling the parameter $n$ we can control the amplitudes of the rational solution $v(x, y, t)$. Moreover, with the change of the parameter $n$, the property of extreme value point does not alter. So, the structure of rational solution $v(t, x, y)$ does not change as the parameter $n$ is varied. Figure 2 shows the profile of rational solution $V(x, y)$. It a typical Peregrine-soliton-like structure, a hump and two holes, two holes separated by a hump. From Figure 2 we can also observe that the profile does not change as the parameter $k$ is varied, except for the increase or decrease of the minimum or maximum values and the slight rotation of the hump orientation.

From the above analysis and numerical simulations, we can obtain that these rational solitary waves are localized in both time and space and decay algebraically to the background plane wave.

\section{Rational Solution of the Standard KP Equation}

The standard KP equation $[2,3,6,7,26]$ can be written as follows:

$$
\left(\psi_{t}+6 \psi \psi_{x}+\psi_{x x x}\right)_{x}+\lambda \psi_{y y}=0, \quad \lambda= \pm 1
$$

It was given by Kadomtsev and Petviashvili $[2,3]$ to discuss the stability of $(1+1)$-dimensional soliton to the transverse long-wave disturbances, which is known as the KP equation. The propagation property of solitary wave solution depends on the sign of $\lambda$ in (16). The coefficient $\lambda$ is defined as follows: $\lambda=1$ for negative dispersion and $\lambda=-1$ for positive dispersion. When $\lambda=-1$, (16) is usually called KP-I, while for $\lambda=1$, it is usually called KP-II.

By the above method we can obtain the rational solitary solution of the standard KP equation:

$$
\begin{gathered}
\psi(x, y, t)=8\left(\frac{1}{\xi^{2}+\eta^{2}-24 / \lambda(q-n)^{2}}\right. \\
\left.-\frac{(\xi+\eta)^{2}}{\left(\xi^{2}+\eta^{2}-24 / \lambda(q-n)^{2}\right)^{2}}\right),
\end{gathered}
$$

where $\xi=x+q y+(1 / 2) \lambda\left((n-q)^{2}-2 q^{2}\right) t, \eta=x+$ $n y-(1 / 2) \lambda\left((n+q)^{2}-2 q^{2}\right) t$, and $q, n$ are real valued constants. This solution is a little different from that given in $[2,7]$. It can be expressed by (17) and can be regard as the two-wave solution about spatiotemporal variable $(x, y, t)$ because of $\psi(x, y, t)=\psi(\xi(x, y, t), \eta(x, y, t))$. From (17), this solution is clearly nonsingular with $\lambda=-1$. It is a nonsingular rational solution. When $\lambda=1$, the denominator of this family of solution has poles. Therefore this solution is not very well behaved. It is a singular rational solution. 
Considering the the maxima and minima for the analytical formulation $\psi(x, y, t)$ with $\lambda=-1, \psi(x, y, 0)$ possesses extreme at

$$
(x, y)=(0,0),\left( \pm \frac{6}{q-n}, 0\right) .
$$

The local maximum is located at $(x, y)=(0,0)$, and the maxima are $(q-n)^{2} / 3$. The local minimum is located at $(x, y)=$ $( \pm 6 /(q-n), 0)$, and the minima are $(q-n)^{2} / 24$. The maxima are eight times as large as the minima. The profile is similar to $v$. As the parameters $q$ and $n$ are varied, the profile does not change.

\section{Conclusions}

In this paper, with the help of the Hirota method and Taylor series (the long wave limits), the integrable Kadomtsev-Petviashvili-based system has been investigated. The breatherlike solution (a pulsating mode) and rational solution are presented. Some very interesting and valuable feature can be observed by changing the wave number of the plane wave. To summarize the theoretical analysis and computer simulation from Section 2.4, we can divide the type of the short-wave rational solution into three cases.

(i) If $\delta^{2} \in(0,1 / 3]$, the function $u(x, y, t)$ shows bright solitary wave feature (see Figure 1(a)).

(ii) If $\delta^{2} \in(1 / 3,3)$, the function $u(x, y, t)$ shows fourlump type solitary wave feature (see Figure 1(b)).

(iii) If $\delta^{2} \in[3,+\infty)$, the function $u(x, y, t)$ shows dark solitary wave feature (see Figure 1(c)).

These analyses also show that the structures of the breatherlike and rational solutions are instable. We perhaps control the the behavior of rational solitary wave by controlling the parameter $\delta$. However, the long-wave rational solution does not possess this feature. Finally, we have discussed the structure of the rational solitary wave solution for the standard KP equation. The singularity of the the rational solitary wave solution depends on the sign of the parameter $\lambda$. It is expected that these results may provide us with some valuable information on the dynamics of the relevant nonlinear sciences fields. Following these ideas in this work, further study may be needed further to see whether system (1) has another type of specially spatiotemporal structure of the solutions such as the higher-order rational solitary wave and how to find them.

\section{Conflict of Interests}

The authors declare that there is no conflict of interests regarding the publication of this paper.

\section{Acknowledgments}

The authors would like to express their sincere thanks to referees for their enthusiastic guidance and help. This work is supported by the National Natural Science Foundation of China (Grant nos. 11301235, 11261049, and 11161025) and the Fund for Fostering Talents in Kunming University of Science and Technology (nos. KKSY201403049 and KKSY201307141).

\section{References}

[1] A. Maccari, "The Kadomtsev-Petviashvili equation as a source of integrable model equations," Journal of Mathematical Physics, vol. 37, no. 12, pp. 6207-6212, 1996.

[2] M. J. Ablowitz and P. A. Clarkson, Solitons, Nonlinear Evolution Equations and Inverse Scattering, Cambridge University Press, Cambridge, UK, 1991.

[3] H. Zhao, "Applications of the generalized algebraic method to special-type nonlinear equations," Chaos, Solitons and Fractals, vol. 36, no. 2, pp. 359-369, 2008.

[4] S. Sheng, "Exp-function method for solving Maccari's system," Physics Letters A: General, Atomic and Solid State Physics, vol. 371, no. 1-2, pp. 65-71, 2007.

[5] A.-M. Wazwaz, "Abundant soliton and periodic wave solutions for the coupled Higgs field equation, the Maccari system and the Hirota-Maccari system," Physica Scripta, vol. 85, no. 6, Article ID 065011, 2012.

[6] H.-L. Zhen, B. Tian, and W.-R. Sun, "Dynamics of an integrable Kadomtsev-Petviashvili-based system," Applied Mathematics Letters, vol. 27, pp. 90-96, 2014.

[7] J. Satsuma and M. J. Ablowitz, "Two-dimensional lumps in nonlinear dispersive systems," Journal of Mathematical Physics, vol. 20, no. 7, pp. 1496-1503, 1979.

[8] N. V. Priya, M. Senthilvelan, and M. Lakshmanan, "Dark solitons, breathers, and rogue wave solutions of the coupled generalized nonlinear Schrodinger equations," Physical Review E, vol. 89, no. 6, Article ID 062901, 2014.

[9] H. N. Chan, K. W. Chow, D. J. Kedziora, R. H. J. Grimshaw, and E. Ding, "Rogue wave modes for a derivative nonlinear Schrödinger model," Physical Review E, vol. 89, no. 3, Article ID 032914, 2014.

[10] G. Mu and Z. Y. Qin, “Two spatial dimensional N-rogue waves and their dynamics in Mel'nikov equation," Nonlinear Analysis: Real World Applications, vol. 18, pp. 1-13, 2014.

[11] Y. J. Shen, Y. T. Gao, D. W. Zuo, Y. H. Sun, Y. J. Feng, and L. Xue, "Non-autonomous matter waves in a spin-1 Bose-Einstein condensate," Physical Review E, vol. 89, no. 6, Article ID 062915, 2014.

[12] D. H. Peregrine, "Water waves, nonlinear Schrödinger equations and their solutions," The Journal of the Australian Mathematical Society. Series B. Applied Mathematics, vol. 25, no. 1, pp. 16-43, 1983.

[13] A. Montina, U. Bortolozzo, S. Residori, and F. T. Arecchi, "NonGaussian statistics and extreme waves in a nonlinear optical cavity," Physical Review Letters, vol. 103, no. 17, Article ID 173901, 2009.

[14] Y. Ohta and J. Yang, "General rogue waves in the focusing and defocusing Ablowitz-Ladik equations," Journal of Physics A. Mathematical and Theoretical, vol. 46, no. 10, Article ID 105202, 2013.

[15] Y. Ohta and J. Yang, "Dynamics of rogue waves in the DaveyStewartson II equation," Journal of Physics A: Mathematical and Theoretical, vol. 46, no. 10, Article ID 105202, 19 pages, 2013.

[16] C. J. Wang, Z. D. Dai, and C. F. Liu, "From a breather homoclinic wave to a rogue wave solution for the coupled SchrödingerBoussinesq equation," Physica Scripta, vol. 89, no. 7, Article ID 075206, 2014.

[17] U. Bandelow and N. Akhmediev, "Persistence of rogue waves in extended nonlinear Schrödinger equations: integrable SasaSatsuma case," Physics Letters, Section A: General, Atomic and Solid State Physics, vol. 376, no. 18, pp. 1558-1561, 2012. 
[18] C. Li, J. He, and K. Porsezian, "Rogue waves of the Hirota and the Maxwell-Bloch equations," Physical Review E, vol. 87, no. 5, Article ID 059903, 2013.

[19] C.-Q. Dai and W.-H. Huang, "Multi-rogue wave and multibreather solutions in PT-symmetric coupled waveguides," Applied Mathematics Letters, vol. 32, pp. 35-40, 2014.

[20] L.-C. Zhao and J. Liu, "Rogue-wave solutions of a threecomponent coupled nonlinear Schrödinger equation," Physical Review E, vol. 87, no. 1, Article ID 013201, 2013.

[21] Z. Y. Yan, "Financial rogue waves," Communications in Theoretical Physics, vol. 375, no. 48, pp. 4274-4279, 2011.

[22] R. Hirota, "Exact solution of the korteweg-de vries equation for multiple collisions of solitons," Physical Review Letters, vol. 27, no. 18, pp. 1192-1194, 1971.

[23] W. X. Ma, "Complexiton solutions to the Korteweg-de Vries equation," Physics Letters A, vol. 301, no. 1-2, pp. 35-44, 2002.

[24] H. Kielhöfer, Bifurcation Theory: an Introduction with Applications to PDEs, vol. 156 of Applied Mathematical Sciences, Springer, New York, NY, USA, 2004.

[25] C. J. Wang and Z. D. Dai, "Dynamic behaviors of bright and dark rogue waves for the $(2+1)$ dimensional Nizhnik-NovikovVeselov equation," Physica Scripta, vol. 90, no. 6, Article ID 065205, 2015.

[26] C. J. Wang and Z. D. Dai, "Breather-type multi-solitary waves to the Kadomtsev-Petviashvili equation with positive dispersion," Applied Mathematics and Computation, vol. 235, pp. 332-337, 2014. 


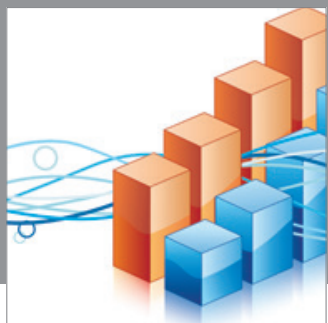

Advances in

Operations Research

mansans

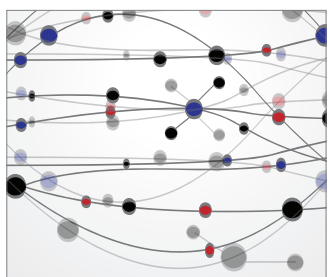

The Scientific World Journal
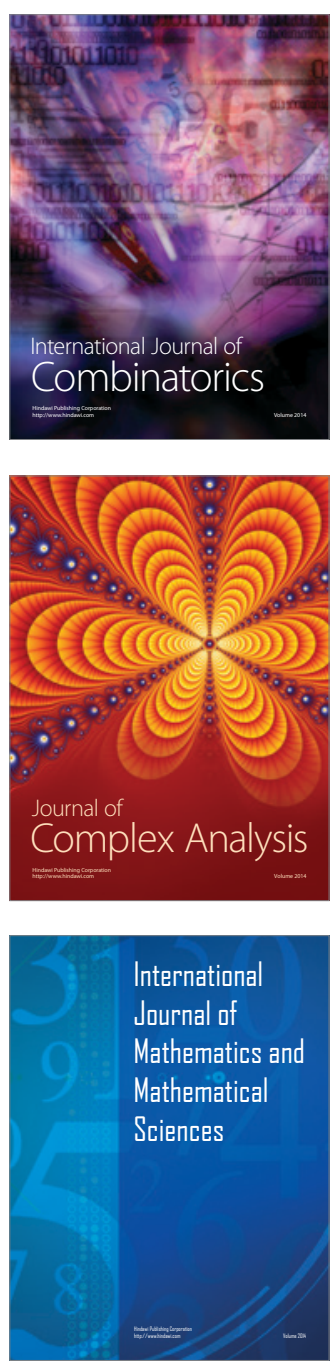
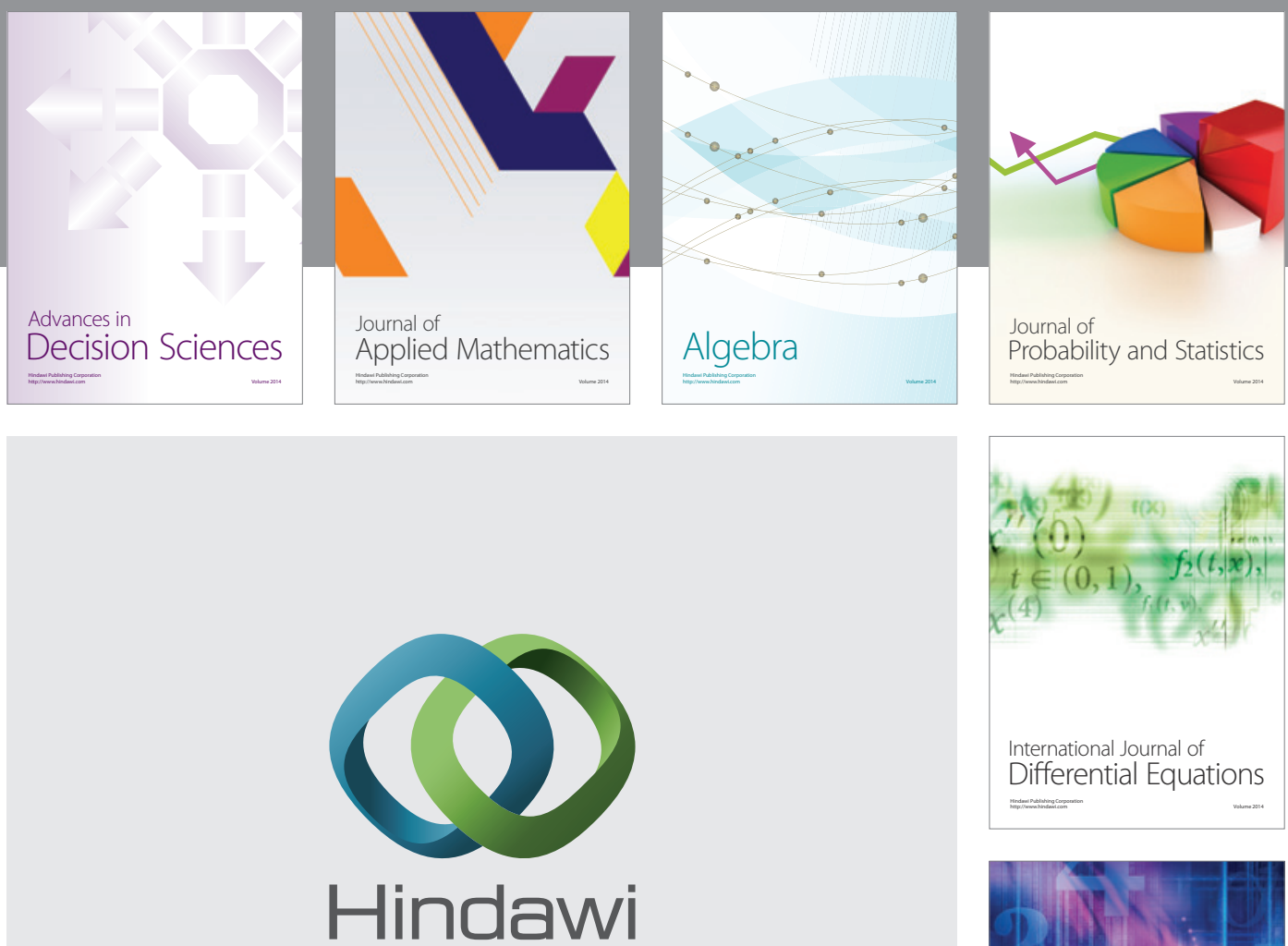

Submit your manuscripts at http://www.hindawi.com
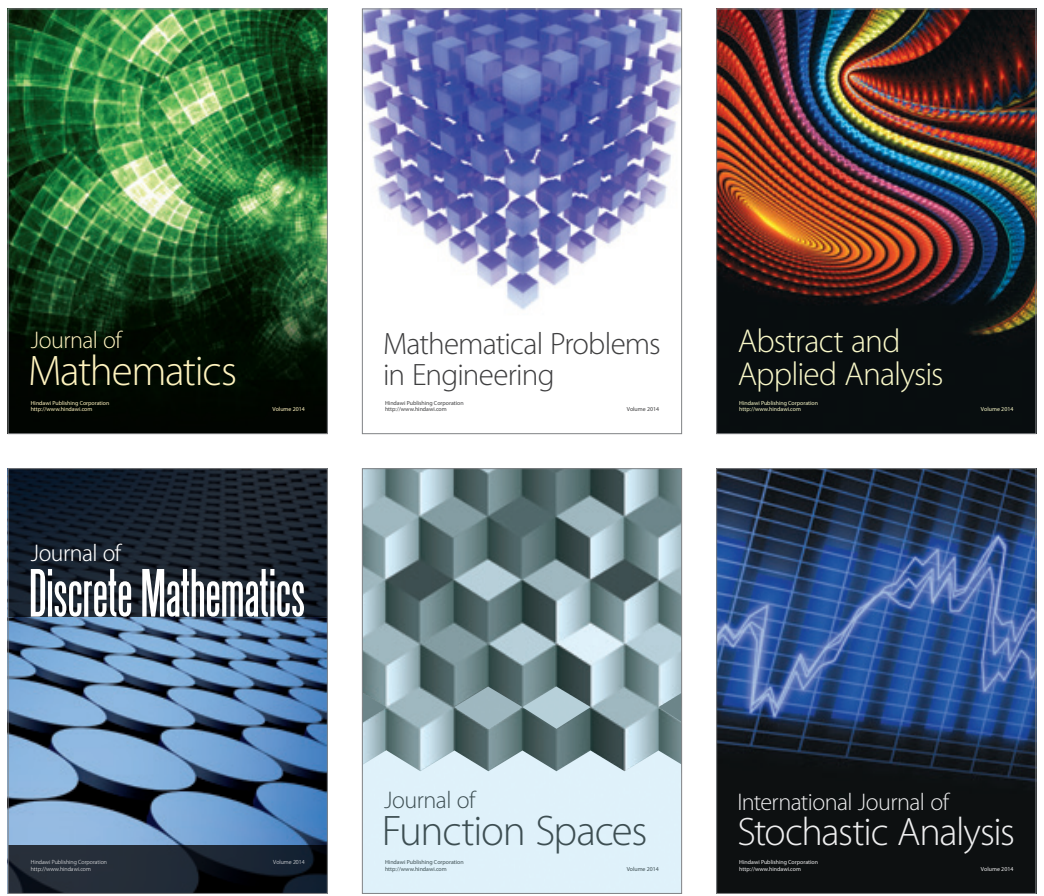

Journal of

Function Spaces

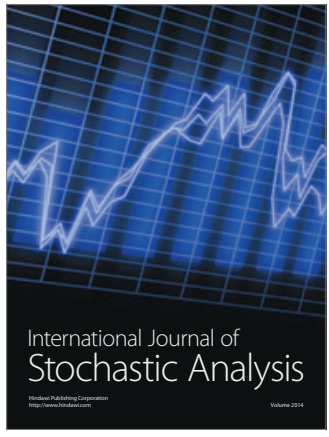

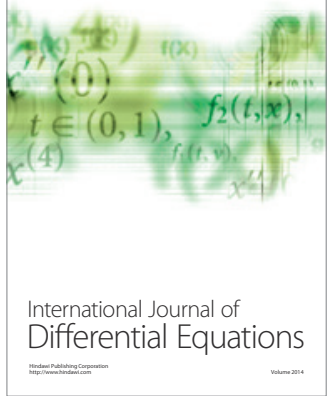
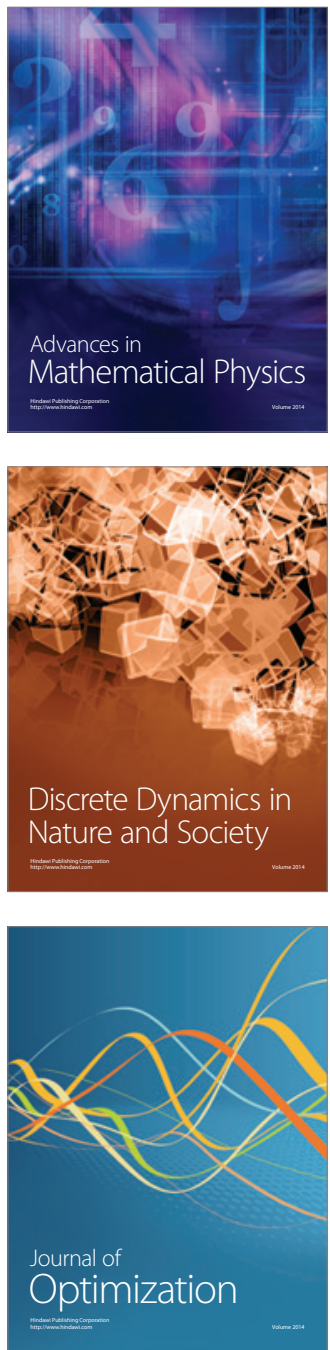